\title{
¿Cómo incentivar la motivación por aprender en nuestros alumnos?
}

MSc. María Catalina Tapia López

Docente investigadora

UNAN-MANAGUA

kathytapia7@hotmail.com

\section{INTRODUCCIÓN}

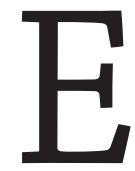

n la práctica docente se manifiesta preocupación por la falta de interés en aprender que muestran muchos alumnos. Lo que lleva a reflexionar y cambiar las estrategias didácticas utilizadas en el aula de clase, para que motiven a los alumnos a mejorar su desempeño académico. Sin embargo, estos cambios no siempre han dado los frutos deseados y se continúa en la búsqueda de esos "puntos claves". El libro "Pedagogía de la Motivación", de Brigitte Prot, permite realizar un análisis más profundo de los procesos pedagógicos que deben ser tomados como puntos de referencia para poder incentivar, día a día, la motivación en los alumnos, ya que la autora a través de esta obra, presenta un conjunto de elementos fundamentales encaminados a despertar el deseo de aprender.

El presente artículo contiene una reflexión acerca de lo que se considera uno de los problemas más significativo que se encuentra en el ámbito académico: los alumnos carecen de motivación por aprender, así como algunos aspectos que han resultado positivos a lo largo de la práctica docente; partiendo de la premisa de que no sirve de nada cualquier cambio que se haga a nivel institucional, curricular, así como cualquier esfuerzo desarrollado por ellos sino incentivan a los alumnos a aprender.

\section{FACTORES QUE INCIDEN EN LA MOTIVACIÓN}

Para poder despertar e incentivar la motivación en los alumnos, se considera importante tomar, como punto de partida, aquellos elementos que inciden de manera positiva o negativa 
en la motivación y sus deseos de aprender. Para ello, se debe iniciar por el núcleo donde el joven crece y se desenvuelve en sus primeros años de vida, hasta llegar a integrarse en una sociedad, en la que los medios de comunicación juegan un rol determinante.

\subsection{Entorno familiar}

La familia es el núcleo fundamental de toda sociedad en el que se inicia la formación del ser humano. Los padres juegan un rol esencial en la educación de sus hijos, en la formación de valores; por tanto, deben ser el incentivo y la piedra angular para hacer de ellos personas de bien que permita la construcción de una mejor sociedad. Cuando el joven crece en una familia donde siempre están pendientes de su formación, de su educación, donde le animan e incentivan a aprender, le ayudan, orientan y escuchan, se encuentran niños y adolescente motivados, seguros de sí mismos, que obtienen buenos resultados en el ámbito académico y social.

Por el contrario, si crece en un entorno familiar donde no es escuchado, no es tomado en cuenta como persona, donde los padres solo se enfocan en satisfacer sus necesidades materiales, creando en ellos una cultura del "sí" como una forma de justificar su ausencia en el hogar, recompensándole a través de cosas materiales, sin limitarles en sus acciones, estos niños y adolescentes no tienen motivación, presentan problemas escolares, debido a que no tienen un horizonte claro hacia dónde dirigirse y se sienten con derecho de hacer lo que se les antoje, sin reconocer ningún tipo de autoridad. Como señala Prot "los padres ya no han dicho "no", o pocas veces, y los jóvenes no han encontrado límites suficientes” (2005, p.30).

Se puede presentar otra situación familiar, como señala Prot, en la que los niños y los adolescentes se sienten valorados solo por sus éxitos, ya sean escolares, deportivos, artísticos o en cualquier otro ámbito. En este sentido, no se sienten reconocidos como personas, como seres humanos (2005, p.62). En este sentido, la motivación de los alumnos no está en el deseo de aprender sino en satisfacer las expectativas de sus padres.

Adicionalmente, se puede presentar otro escenario donde los niños y adolescentes se encuentran sobre asistidos y muchas de sus asignaciones académicas son realizadas por su "personal de apoyo" que lo asesora (o le colabora) fuera del aula de clase. Esta situación no les permite desarrollarse, académicamente, sus tareas le son "hechas", presentándose entonces con dos situaciones extremas que, de una u otra forma, no crean las condiciones que permiten incentivar la motivación por aprender y surge la necesidad de encontrar un poco de equilibrio,

"se trata de encontrar un punto medio entre dos comportamientos extremos de adultos que encontramos hoy: o bien, los niños se encuentran abandonados a su suerte, o bien, están sobre asistidos" (Prot, 2005, p. 62). 
Se debe tener presente que, "una persona asistida pierde algo de su deseo propio y de su autonomía: el asistencialismo crea una deficiencia personal" (Prot, 2005, p.11). Cuando un alumno sobre asistido llega al ámbito universitario, suele ocurrir que busca asistencia o colaboración en este nivel académico, pero no siempre lo hace por los motivos correctos. Su "búsqueda de ayuda" no está incentivada por sus deseos de aprender, sino por la necesidad de obtener una calificación que le permita aprobar la asignatura. A continuación, se ejemplifica lo expuesto de la siguiente manera:

Al enseñar la asignatura de Programación a un grupo de alumnos de segundo año de la carrera de Ciencias de la Computación, en una ocasión se les asignó la elaboración de un conjunto de programas que daban respuesta a una serie de problemas concretos. Para comprender la lógica que habían utilizado se decidió que, uno a uno, frente a la computadora, explicara paso a paso de qué manera había organizado la solución. Con esta actividad se constató que ni el $50 \%$ de los alumnos había trabajado en los programas, sino que se los habían "encargado" a alumnos de año superiores. Esto porque sentían más la necesidad de aprobar la asignatura, que aprender la solución de los casos asignados. Esto confirma la necesidad urgente de incentivar en los estudiantes un deseo real de aprender.

\subsection{Entorno social y la influencia mediática}

Se vive en una sociedad del consumismo, carente de valores, de referentes, con pocos líderes que sirvan de "inspiración" a los estudiantes. Esta sociedad se encuentra inmersa en actitudes de pasividad y conformismo.

"Nuestra sociedad es imitadora. No hay creatividad, nos enganchamos a un pasado que ya no tiene sentido, sin duda porque no llegamos a construir el presente sin imaginar el futuro" (Prot, 2005, p.30).

Esta situación influye de manera negativa en nuestros alumnos, les hace perder el sentido del por qué o para qué estudiar si al final van a engrosar la lista de profesionales que están en el desempleo, o bien, desempeñar cargos para los que se requiere un mínimo de preparación académica. Esto genera que los alumnos pierdan su interés por desarrollarse académicamente y que poco a poco vayan abandonando las aulas de clases. O bien, culminen la carrera "por qué no hay opción" para satisfacer a sus padres, o porque la sociedad dicta que se debe tener un título universitario para "ser alguien en la vida". Estos alumnos no tienen la motivación de aprender, sino de aprobar para alcanzar una meta que al final, no tiene un significado real para ellos. Provoca una "huida de una realidad demasiado pesada para cargar con ella, la de un contexto socioeconómico que, a priori, les ofrece pocos espacios" (Prot, 2005, p.62).

La influencia de los medios de comunicación es significativa en la falta de motivación de los alumnos, quienes viven "bombardeados" de mensajes en los que se valoran a las personas 
por lo que tienen y no por lo que saben; donde se da "el abandono del ser, el culto al tener, esta cultura de la apariencia y del individualismo nos lleva a la catástrofe y a una increíble pasividad" (Prot, 2005, p.28).

Este culto por el tener y el abandono del ser provoca un desinterés, casi generalizado, por el deseo de aprender, de conocer, de saber. Prot expresa, que estamos ante una generación que hace de nuestros niños y adolescentes el banco del consumo (2005, p.28), lo que provoca que su motivación principal sea obtener un empleo que le permita generar ingresos, para así poder satisfacer sus necesidades de consumismo desenfrenado en su afán por encontrar su identidad.

\section{A NIVEL EDUCATIVO, ¿CÓMO ESTÁ LA MOTIVACIÓN?}

\subsection{Docencia: Una profesión poco valorada}

En nuestro entorno, al igual que en otras partes del mundo, la docencia es una profesión que se encuentra, en cierta medida, subvalorada, tanto a nivel económico como profesional, en todos los niveles académicos. Esta situación se agudiza en primaria y secundaria, en que muchos docentes se ven en la necesidad de trabajar en turnos dobles para poder hacer frente a una difícil situación económica.

Lo anterior, unido a la falta de motivación por no sentirse valorados, trae como consecuencia que la gran mayoría de los docentes se preocupen, principalmente, por impartir sus clases y cumplir con el programa de la asignatura, en la cual, sus estrategias didácticas son muy pobres y limitadas, que no incentivan ni motivan a sus estudiantes a aprender a pensar, a analizar, a descubrir. En muchos casos, se trata de una educación "tradicionalista", en el que el docente es el eje central de clase y los alumnos se limitan a ser receptores, para luego "reproducir" lo que sus docentes les han "enseñado".

Entonces, se presenta un escenario donde no se brinda a los alumnos las herramientas que les enseñen cómo dar respuesta a una situación del entorno. Estos "vacíos" repercuten significativamente en el rendimiento, en su autoestima y, como consecuencia, en su motivación por aprender. Llegan al siguiente nivel educativo prácticamente "desarmados". Como señala Prot, "un alumno que no haya adquirido métodos de trabajo en primaria, por más que se despierte y ponga interés después, tendrá que adquirirlo de una u otra manera para salir adelante" (2005, p.49).

\section{2. ¿Se verifica si los alumnos están aprendiendo?}

Pocas veces el docente se cuestiona si los alumnos realmente están aprendiendo, lo único que hace es, llegar al aula, desarrollar la clase y dar por cumplida su labor. Pero, ¿cuántas veces él se detiene a lo largo de la clase y, con un verdadero interés, pregunta al estudiante si está 
comprendiendo? A continuación se presenta un caso que nos muestra el comportamiento de nuestros alumnos cuando el docente verifica lo aprendido:

“Enseñaba la asignatura de métodos numéricos a estudiantes de la carrera de computación. En el programa de esta asignatura se abordan un conjunto de técnicas para resolver problemas formulados mediante modelos matemáticos, de manera particular, una de estas técnicas presenta mayor grado de dificultad. En una ocasión me encontraba explicando ante un grupo y al momento de consultarles si me había comprendido, contestaron afirmativamente, sin embargo, no sentí convencimiento en ellos. Desarrollé otro ejemplo y les volví a consultar. Su respuesta siempre fue afirmativa, pero el nivel de convencimiento no varió. Entonces les dije: “Tomen una hoja, escriban su nombre, van a resolver un ejercicio utilizando está técnica. Lo voy a evaluar en 10 puntos". Esta frase provocó la reacción de mis alumnos, en ese momento, sentí que "despertaron" y comenzaron a expresar que habían comprendido parcialmente" (la autora).

Muchas veces el docente no se detiene a preguntar: ¿Mis alumnos comprenden lo que les estoy enseñando? ¿Mis estrategias pedagógicas están funcionando? ¿La forma en que imparto mi clase motiva a mis alumnos a aprender? Estas preguntas servirían como guía para que el docente reflexione sobre las prácticas impartidas en el aula de clase.

\subsection{Zona de comodidad}

En docencia universitaria se ha constatado que algunos docentes que por años han impartido la misma asignatura, en diferentes carreras, llegan al aula con el mismo material que han utilizado por más de 5 años. Se encuentran situados en su "zona de comodidad", que muestran falta de interés por brindar a sus alumnos una educación que vaya acorde a sus necesidades y que esté orientada al perfil de la carrera en la que se están formando.

En su mayoría, son profesionales que se han dedicado a la docencia porque no han encontrado opciones en su campo laboral, no poseen una formación pedagógica y tampoco se preocupan por tenerla. Desarrollan su clase retomando, quizás, estrategias utilizadas por los docentes que les impartieron clase a ellos; no se preocupan por actualizarse a nivel científicotécnico y cuando se les convoca a recibir capacitaciones pedagógicas y metodológicas, que les permita reflexionar sobre su práctica y conocer nuevas estrategias didácticas, asisten por cumplir con una obligación, no con la intención de incorporarla en el aula de clase.

Sin embargo, se presenta otro escenario de docentes, que en solitario, realmente se preocupan por la formación de sus alumnos, que innovan en el aula de clase, que investigan de manera constante a fin de enseñar a sus estudiantes conocimientos técnicos actualizados, que dan asesoría, que vinculan la teoría con la práctica. Sin embargo, estos esfuerzos individuales, no siempre logran incentivar el deseo de aprender en los alumnos. 


\section{4. ¿HACIA DÓNDE SE DEBE IR?}

Para poder determinar hacia dónde se debe ir, qué cambios se deben realizar, es necesario hacer un alto en el camino y reflexionar acerca de la labor docente. Esta labor debe desarrollar acciones que permitan incentivar en los alumnos el deseo de aprender, de conocer, de saber. Se debe "tomar gusto" a lo que se hace, comprometerse con esta misión y estar consciente que, en gran medida, depende de la actuación en el aula de clase, el éxito o el fracaso de los alumnos.

En este punto, vienen a la mente dos preguntas esenciales: ¿Qué cambios se deben hacer como docentes para ser capaces de incentivar la motivación? ¿Cómo se puede incentivar la motivación por aprender en los alumnos?

\subsection{Docentes capaces de motivar}

El papel que desempeñan los docentes, tanto dentro como fuera del aula de clase, es fundamental para poder incentivar la motivación. Hay que recordar que "la motivación de los alumnos pasa por la de los profesores" (Prot, 2005, p.20). Es decir, si no se está motivado como docente, jamás se tendrá la capacidad de motivar a los alumnos.

Conviene renovar la práctica, desarrollar la escucha, estar consciente de la importancia de investigar, de estar actualizados, de incentivar en los alumnos un pensamiento crítico, analítico, reflexivo, que le permita tomar el sentido del porqué y para qué aprender. A continuación se detallan algunos de estos elementos que todo docente deberá mejorar para poder motivar.

\subsubsection{Cambiar la práctica pedagógica}

Para incentivar la motivación se debe transformar la práctica pedagógica, a fin de ayudar a los alumnos a encontrar el sentido de su presencia en clase. Al respecto, Prot externa que, se debe salir del esquema tradicional y situar al alumno, y su acto de aprendizaje, en el centro de la práctica docente (2005, p.109).

Se deben crear escenarios donde el alumno sea el protagonista principal del proceso de enseñanza-aprendizaje y el docente juegue el rol de guía, de orientador, de motivador. Como señala Prot, "el punto de anclaje de una nueva motivación lo constituye el nuevo lugar que tiene que adoptar el educador" (2005, p. 109).

Esto implica llevar a cabo una revisión de los programas de asignaturas y ajustarlos acorde a las necesidades de los alumnos según su perfil profesional. Se debe dar un sentido a la teoría que se desarrolla para que ellos comprendan la utilidad de este conocimiento técnico y puedan determinar cuándo y de qué forma aplicarlo. "Las clases, hoy, necesitan 'personas de campo', que se atrevan a inventar nuevas herramientas y a adaptarse a las necesidades" (Prot, 2005, p.109). 
En definitiva, hay que cambiar la práctica pedagógica, trabajar de otra manera y esto significa "tener presente en el corazón y en la cabeza dos ejes diferentes: la clase y el alumno en su identidad de trabajo, de resultados y de comportamiento, con prioridades diferentes para cada uno" (Prot, 2005, p. 14).

\subsubsection{Aprender a comunicarse}

Es primordial, en la práctica docente, la comunicación adecuada con los estudiantes, del poder que tiene la escucha y el respeto a las opiniones de los demás. Cuando un alumno es escuchado, siente que es valorado, que se le toma en cuenta, elevándose así su autoestima y, por consiguiente, su motivación.

Cuando la comunicación es adecuada, se crean espacios donde, alumnos y docentes, se sientan respetados tanto en su identidad como en el lugar que ocupan en la clase (Prot, 2005, p.105). La forma en cómo se da la comunicación y se expresan los alumnos determina, en gran medida, el clima que se genera en el aula de clase. Si se muestra apertura, respeto, cordialidad, si desarrolla la escucha y generan espacios para que los estudiantes pueden expresar su opinión, se crea un clima de confianza, un espacio donde son respetadas y escuchadas sus ideas, opiniones, sus dudas y demás. De esta manera, como indica Prot "el educador instituye un espacio relacional ajustado a cada clase, fija las 'reglas del juego' y deja abierta la posibilidad, para todos, de comunicarse con él, con su propio yo" (2005, p.106).

Estos espacios de comunicación, incentivan de manera significativa el deseo por aprender, ya que el docente da apertura a que expresen sus dificultades y así poder aclarar dudas y darle un sentido a lo que están aprendiendo. Como señala Prot, "hay que dar una respuesta de manera que el alumno se percate que ha sido escuchada su petición" (2005, p. 107).

En este sentido, el iniciar muy joven la práctica docente, se presentan situaciones en la que los alumnos son cinco o seis años menores. Ante esto, y por la falta de experiencia, se considera mantener la distancia con ellos para "darse a respetar", esto no genera espacios para el diálogo, para la escucha y se mantiene una actitud muy seria en el aula de clase que no coincide muchas veces con la personalidad del docente y genera, en cierta medida, un clima de tensión. En las clases, la participación no es voluntaria, los alumnos temen expresar sus ideas. Son los colegas quienes valoran la actitud del docente al comentar que él es temido por los alumnos, por su actitud seria y estricta. Este comentario obliga a hacer un alto en el camino y reflexionar, que solo el diálogo franco y abierto con los alumnos, en un marco de respeto permite ser muy queridos por ellos y a no ser "temidos".

Entre mayor sea la participación de los alumnos en el aula de clase, más enriquecedor y motivador será el proceso de aprendizaje. Al respecto, Prot señala, que "esta comunicación con 
las múltiples interacciones, determina la calidad de la situación de aprendizaje y constituye, como ya se ha visto, el incentivo central de la motivación de los alumnos" (2005, p. 108).

Se debe tener siempre presente que "la comunicación y lo relacional son herramientas de motivación gracias a las cuales transmitimos conocimientos. No hay que olvidar jamás el fin que se persigue" (Prot, 2005, p.19).

En este punto de la comunicación, es importante y significativo que se agregue un poco de humor durante el desarrollo de la clase, es un elemento que permite "romper el hielo" y crear un clima de confianza con los alumnos, que los incentiva y los motiva a asistir a la clase. Además, como señala Prot, "cuando integramos el humor en nuestro trabajo, ganamos en humildad como maestros, y entonces nos encontramos con la humanidad de los alumnos" (2005, p.17).

\subsubsection{Abandonar el trabajo en "solitario"}

La práctica pedagógica puede mejorar y enriquecerse de manera significativa cuando se comparten las experiencias, aciertos y errores con otros maestros, mediante la implementación de equipos pedagógicos, que se constituyan como los "verdaderos gestores del cambio" (Prot, 2005, p.46).

Todas las innovaciones pedagógicas que han dado valiosos resultados en el aula de clase, deben ser compartidas, como indica la autora, "las iniciativas pedagógicas: deben ser catalogadas, difundidas y darse a conocer más de lo que se está haciendo" (Prot, 2005, p.47). Es por ello que, se considera de vital importancia abandonar el trabajo en solitario y comenzar a conformar equipos pedagógicos que permitan diseñar escenarios que incentiven en los alumnos el deseo por aprender.

Muchas veces, cuando el docente abandona el trabajo en solitario permite incentivar la motivación por aprender en los alumnos. "En mi práctica docente me he desempeñado en las carreras de Ciencias de la Computación e Ingeniería en Sistema. Una de las asignaturas que imparto está orientada al análisis y el diseño de sistemas de información. En algunas ocasiones no he trabajado en solitario, sino que me he coordinado con el docente de programación, a fin de proponerle a los alumnos un solo proyecto de curso, la motivación en ellos se incrementa de manera sorprendente, porque les permite vivir la experiencia de ver concretizado su esfuerzo, su aprendizaje, en un producto específico, en este caso particular, en un sistema de información creados por ellos y que trabaja eficientemente" (la autora).

\subsubsection{Incorporación de las nuevas tecnologías}

Los alumnos son nativos digitales, para ellos, el uso de la tecnología forma parte de su vida cotidiana. Prot plantea que, integrar las nuevas tecnologías en la enseñanza es un paso 
obligatorio y que existen tres razones para esto, una de ellas es la familiarización y cercanía de los alumnos con estas herramientas (2005, p. 116).

Los alumnos se entusiasman cuando un docente hace uso de las redes sociales, de manera particular de Facebook, como una alternativa de comunicación fuera del aula de clase. En ese entorno, ellos escriben sus comentarios y consultas, que son retroalimentados por el maestro y demás compañeros, incentivando así, sus ganas de aprender. Como indica Prot "permite una mediación de los aprendizajes muy interesante" (2005, p.116).

Además de las Redes Sociales, existen otros entornos virtuales de aprendizaje diseñados especialmente para el ámbito académico. Es importante señalar, que los mecanismos que permitan su inserción significativa de las tecnologías, en el proceso de enseñanza-aprendizaje dependen, en gran medida, de las estrategias diseñadas por el docente, así como del currículo educativo.

\subsection{Motivar a los alumnos}

Prot señala, que un factor que impide que un alumno se encuentre motivado, es su falta de confianza en sí mismo (p.16, 2005). Y esta falta de confianza, muchas veces, viene asociada por haber experimentado el fracaso, la no consecución de sus logros, o bien, por no sentirse valorado ni escuchado como ser humano. La motivación está vinculada con la autoestima, en este sentido, Prot indica, que "nace de la capacidad de creer en uno mismo" (2005, p.16).

Se debe buscar la forma de cómo motivar a los alumnos. Prot externa que "la motivación no viene, en primer lugar, de la mente o de la razón, sino que, aunque parezca imposible, viene de las entrañas" (2005, p.16), es decir, debe surgir del interior de cada uno, es por eso que, el primer paso que se debe realizar, es guiar a los alumnos en la búsqueda de su propia motivación. Sin embargo, a como indica Prot, en el ámbito académico, esta búsqueda debe estar acompañada por un sentido, debe haber un para qué y por qué hacerlo.

Este sentido, se puede alcanzar proponiéndole el desarrollo de un proyecto académico, orientado a un área de interés en particular, sin perder de vista los objetivos de la asignatura. Lo ideal es definir, de manera conjunta con los alumnos, el alcance de este proyecto, tomando como referencias sus fortalezas y sus puntos débiles. Como señala Prot, se deben "definir objetivos accesibles, las etapas y los medios necesarios para alcanzarlos y situarlos en el tiempo" (2005, p. 72).

Con la práctica docente se constata cómo cambia el interés y la motivación de los alumnos, cuando se les involucra en la toma de decisiones referidas a los trabajos de fin de curso. Muchas veces los docentes elaboran y orientan los proyectos sin tomar en consideración la opinión de ellos y no se toma el tiempo para analizar si el trabajo asignado era o no de su agrado. 
Para incentivar la motivación, deben ser los alumnos quienes propongan el trabajo que les gustaría realizar, siempre en el marco de los objetivos de la asignatura. Debe revisarse con cada grupo su propuesta y definir las herramientas a utilizar y la línea de trabajo a seguir. Este cambio de estrategia permite reducir el porcentaje de reprobados, ayuda a combatir su indiferencia por aprender y que asuman, con responsabilidad, su rol en el proceso de aprendizaje. Al respecto, Prot expresa, que definir bien los objetivos, el lugar de los alumnos y sus responsabilidades, permiten reducir decepciones (2005, p.113).

Una vez que se logra crear la motivación de aprender en los alumnos, a través de un proyecto o trabajo académico, es vital brindarles un proceso de acompañamiento y asesoría, para así poder mantener viva esa llama, "se ayudará al alumno a motivarse haciendo una revisión regular de su situación escolar” (Prot, 2005, p. 72). Y durante ese acompañamiento, felicitarlo por los logros alcanzados, como señala Prot, "validar los éxitos en cada recorrido, más que llevar a cabo una evaluación basada en los errores" (2005, p. 73).

\subsubsection{Ayudarles a encontrar sus fortalezas}

Hay asignaturas que cuestan más que otras. En definitiva, todos somos buenos en algo. Algunos tienen más habilidad para la matemática, otros para las ciencias, otros para las artes, otros para la música, entre otros. Para poder incentivar la motivación, se debe ayudar a los alumnos a encontrar cuáles son sus puntos fuertes y a detectar sus dificultades.

Se debe descubrir con ellos el método de trabajo que les resulte mejor y, como señala la autora, hay que tener presente que "educar a un alumno es partir de su potencial, para que crezca, se construya, se desarrolle" (Prot, 2005, p. 45).

Bajo ninguna circunstancia se debe comparar el desempeño de un alumno con el de otro, no hay nada más desmotivante que sentirse menos inteligente o menos capaz que sus compañeros. Por el contrario, se debe proponerle retos personales, que permitan "ayudarles a competir entre ellos mismo y a superarse" (Prot, 2005, p. 68).

\section{CONCLUSIONES}

Como docentes, se debe tener la misión y obligación de crear las condiciones y aplicar estrategias pedagógicas que incentiven el deseo de aprender, de saber, de conocer; debe dársele un sentido de aplicación práctica a los contenidos teóricos que se desarrollen. El eje central que determina el éxito o el fracaso de los alumnos, es la motivación que ellos tengan por aprender, lo que le permitirá enfrentarse a los retos del entorno.

No debe perderse de vista, que cada alumno se motiva de forma diferente y que debe haber experimentado en algún momento de su vida, la sensación del éxito para poder motivarse. La 
motivación debe surgir desde lo más profundo de su ser, de lo contrario, todo esfuerzo realizado por los docentes, no tendrá el efecto deseado.

Debe diseñarse estrategias didácticas que permitan dar respuesta a las necesidades de los alumnos, impulsando la formación de equipos pedagógicos que permitan enriquecer la práctica docente. Debe estarse constantemente actualizado en el área de especialidad y con una mente abierta al cambio.

Es de vital importancia mantener una comunicación efectiva con los alumnos, que se sientan escuchados y valorados. Debe crearse un clima en el aula de clase que invite al análisis, a la reflexión, colocando al alumno como eje central de su proceso de aprendizaje, dotándolo de las herramientas metodológicas que le faciliten la adquisición de conocimientos.

La lectura de esta obra lleva a una reflexión profunda sobre aspectos esenciales que deben ser tomados en cuenta para lograr incentivar la motivación y las ganas de aprender, aspectos que de ahora en adelante se deben poner en práctica mediante diversas estrategias y herramientas metodológicas, que se ajusten a las necesidades y características de cada grupo de clase.

\section{BIBLIOGRAFÍA}

Prot, B. (2005). Pedagogía de la motivación. Cómo despertar el deseo de aprender. Madrid: Narcea, S.A. de Ediciones. 